\title{
Dot Diffusion Block Truncation Coding for Satellite Image Retrieval
}

\author{
Deepti Chavan \\ P.G Student \\ Department of CMPN, \\ Thakur College of Engineering \& Technology \\ Kandivali (E) Mumbai
}

\author{
Kiran A. Bhandari \\ Associate Professor \& Deputy H.O.D \\ (Computer Dept.), \\ Thakur College of Engineering \& Technology \\ Kandivali (E), Mumbai
}

\begin{abstract}
In present survey it is noticed that the profound interest in research and study of retrieval of satellite images and Image Retrieval on Content Based is grown hugely .Thus, to build the semantic error which is a huge challenge. Also, it prevents wide hosting of image on content based search engines which is now the necessity of CBIR technique. Mostly image search engine depend on human generated data as input query namely tags or annotations. The images get stored in database according to the annotations or tags assigned to them. Thus, tags or annotations are used as inputs by search engines.Thus, to overcome the limitation of annotations based image retrieval an Improved Block Truncation Coding is used which is based on Texture and color features of an image. In this paper, Improved Block Truncation Coding technique is used to fill the semantic gap. Then the similarity features type of search is carried to find different query regions namely desert, coastal, forest, metro from the satellite images. The retrieval technique can be practically applied and compared using different similarity methods .The technique of Improved Block Truncation Coding is described which is one of the most proficient methods used in retrieval of satellite images. Though, in traditional BTC certain redundancy like false contour, inherent artifacts are observed. In order to deal with this an improved BTC with dot- diffusion is applied to the system. Here, a Dot Diffusion is added for retrieving the most relevant images that best match to the query image whereas Dot Diffusion is used to give best quality image with good clearity and save memory need to store in database.
\end{abstract}

\section{Keywords}

Improved Block Truncation Coding (IBT Color,) Image Retrieval, Block Truncation Coding (BTC), Satellite Image Retrieval (SIR), Dot Diffusion Block Truncation Coding (DDBTC).

\section{INTRODUCTION}

Block truncation coding (BTC) is basically an image compression technique which was shown and proved to us by Delp and Mitchell in 1979 [1]. Block truncation coding (BTC) is a moreover a lucid image coding technique which was found developed in the early years of digital image processing. This is proved to be quick image compression technique which achieved a bit rate of 1.999 bits per pixel nearly rounding to 2.0 bits per pixel. BTC is not only used for coding or processing the satellite image, but it can also be very efficiently and effectively used in satellite image indexing and retrieval for image dataset or database application for grey scale image methods [10] and satellite image retrieval. The basic work flow of this technique is to partition the original image into many non-overlapped blocks and each of which is represented by two unlike values namely, mean and standard deviation.. Mostly in every CBIR system, features are the only thing to represent the content or the details of the image. There are only three types of features like color, texture and shape which can be considered for research work. The combination of both of the features firstly, the Text or Tags Based Image Retrieval and secondly, Content Based Image Retrieval has developed representations of images more effective [2]. The major aim of image retrieval system is to retrieve best matched images as possible from loaded dataset of satellite images such that the retrieved resultant images meet the user's requirement.

The requirements of the user can be displayed in terms of similarity to that of the other images or in terms of features matching that depend on what type of CBIR system is used . The system aids the user to access, browse and also retrieve images efficiently and possibly in real time, from those satellite image databases. Image retrieval systems can be learnt into two types: Text Based Image Retrieval and Content Based Image Retrieval. The Content based retrieval is of three types: Content based image retrieval, Content based audio retrieval, and Content based video retrieval.

Here in the paper image retrieval is explored considering feature like texture and color of the satellite image. Firstly, the texture and color features of the image is extracted by applying the BTC technique .Secondly, the improved BTC technique is applied on it and satellite image is retrieved lastly dot diffusion is used at the time of relevant feedback.

The following paper is summed up as below: Section II shows the related research done. Section III shows the Block Truncation Coding. Section IV indicates the Proposed Improved Block Truncation Coding. Section V displays experimental results and Section VI draws towards conclusion.

\section{RELATED WORK}

Authors M. Mese and P. P. Vaidyanathan, analysed Optimized half toning using dot diffusion and methods for inverse half toning [1].They have incorporated BTC to yield the proposed dot-diffused BTC (DDBTC) technique. Here cooptimization of both diffused and class matrix are obtained for even better image quality as compared to that of EDBTC, Also it has maintained the technique of parallelism which is similar to that of ODBTC. The two features describe the proposed DDBTC can be efficient compression applications and low power systems for image/video recording. Thus, the proposed method can be considered as a powerful technique 
for to use in most of the low power image/video codec system.

Authors H.B. Kekre, S.D. Thepade et al. analysed Image Retrieval with Shape Features extracted using Gradient Operators and Slope Magnitude technique with BTC [4]. They have considered generic image dataset of 1000 images of 11 different categories and tested it. The performance analysis is studied upon the average precision and recall computed of all queries. Mask Shape type of BTC technique is used but it has limitation is that the query image and database image should be of the same size. Also, Operator like Gradient is used for extraction of shape feature. Operators like Prewitt, Robert, Sobel and Canny are used for shape extraction features.

Authors Y.N.Mamatha and A.G Ananth have described CBIR of Satellite Imageries using Soft Query Based Color Composite Technique [5].The authors suggest that color can be used as data or content. Therefore, content based image processing was carried out for sample dataset of satellite images consisting of urban image of high resolution and rural image of low resolution.

Authors Yu-f-x, Luo, H, Lu, Z-m have described Color image retrieval using pattern co-occurrence matrices based on BTC and Vector Quantization[6].They explained each color image input is decomposed into $\mathrm{Y}, \mathrm{Cb}$ and $\mathrm{Cr}$ components and then the technique of BTC is applied to the $4 \times 4$ blocks of Y. The pair of mean and bit plane sequences are quantized with the contrast pattern codebook and visual pattern codebook in order to obtain the contrast and visual pattern co-occurrence matrix. Vector Quantization is applied to $4 \times 4$ blocks of $\mathrm{Cb}$ and $\mathrm{Cr}$ blocks with the $\mathrm{Cb}$ codebook and $\mathrm{Cr}$ codebook respectively. Thus, color pattern co-occurrence matrices are received. These Retrieval simulation output is compared with existing two BTC-based features, highly improve retrieval efficiency.

Authors H.B. Kekre, S.D. Thepade have described Image Retrieval using Augmented Block Truncation Coding Techniques [11]. They showed the Binary block truncation Coding that is based on the color features of the CBIR methods of the image. This approach recommends red, green and blue planes of image together in order to compute the feature vector. Also augmentation has been done on the BTC based CBIR as BTC-RGB and Spatial BTC-RGB. In the BTC-RGB the feature vector is produced by considering planes such as red, green and blue of the images independently. While that in Spatial BTC-RGB the feature vector is composed of blocks of four. Each block is explaining the features extracted from one of the four non overlapping quadrants of the image. This method is tested on the thousand images dataset and the results describe that the precession is improved in BTC-RGB and is even more better in Spatial BTC-RGB.

\section{PRIMITIVE BLOCK TRUNCATION CODING}

Block Truncation Coding or BTC is a lossy image compression technique. It is applied to grayscale images in 2D type. Here, below the procedure explains that first the preprocessing of the image is done and then the mean followed by standard deviation is found and thus image is compressed.
Procedure:

A $256 \times 256$ pixel image is divided into blocks of $4 \times 4$ pixels. Mean and Standard Deviation are calculated for each block. Quantization is performed on block as follows:

$$
y(i, j)=\left\{\begin{array}{l}
1, x(i, j)>\bar{x} \\
0, x(i, j) \leq \bar{x}
\end{array}\right.
$$

Where $(i, j)$ are pixels elements of the original image block .$y(i, j)$ are elements of compressed block.

If a pixel value is less than the mean it is assigned the value " 0 " otherwise " 1 ".

The 16 bit block is stored or transmitted along with the values of mean and Standard Deviation. Then reconstruction is done with two values "p" and "q" The values of "p" and "q" can be computed as follows:

$$
\begin{aligned}
& p=\bar{x}-\sigma \sqrt{\frac{a}{m-a}} \ldots \ldots \ldots \ldots \ldots \ldots(1.2) \\
& q=\bar{x}+\sigma \sqrt{\frac{m-a}{a}} \ldots \ldots \ldots \ldots \ldots \ldots(1.3)
\end{aligned}
$$

Where $\sigma$ is the standard deviation, $\mathrm{m}$ is total number of pixels in the block and a is number of pixels greater than mean $(\bar{x})$

To, reconstruct the image, elements those assigned " 0 " or "1"are replaced with "p" and "q" respectively.

$$
x(i, j)=\left\{\begin{array}{l}
p, y(i, j)=0 \\
q, y(i, j)=0
\end{array}\right.
$$

\subsection{Proposed Improved Block Truncation Coding Using Dot Diffusion}

The Fig.1 explains:

1. Here the query image is fired by the user.

2. As soon as the query image is fired the preprocessing of the image is done. In preprocessing the query image is resized, colour $2 \mathrm{rgb}$ conversion is done.

3. The texture and color features are retrieved of the image.

4. The BTC is applied on the images features and most relevant image is retrieved.

5. The image features are matched to that of feature vector database.

6 . To get the only most relevant image the similarity measurement is performed by Euclidean distance.

7. Then a threshold is kept for the relevant result images and Dot Diffusion is applied on it.

8 . Thus the result is obtained. 


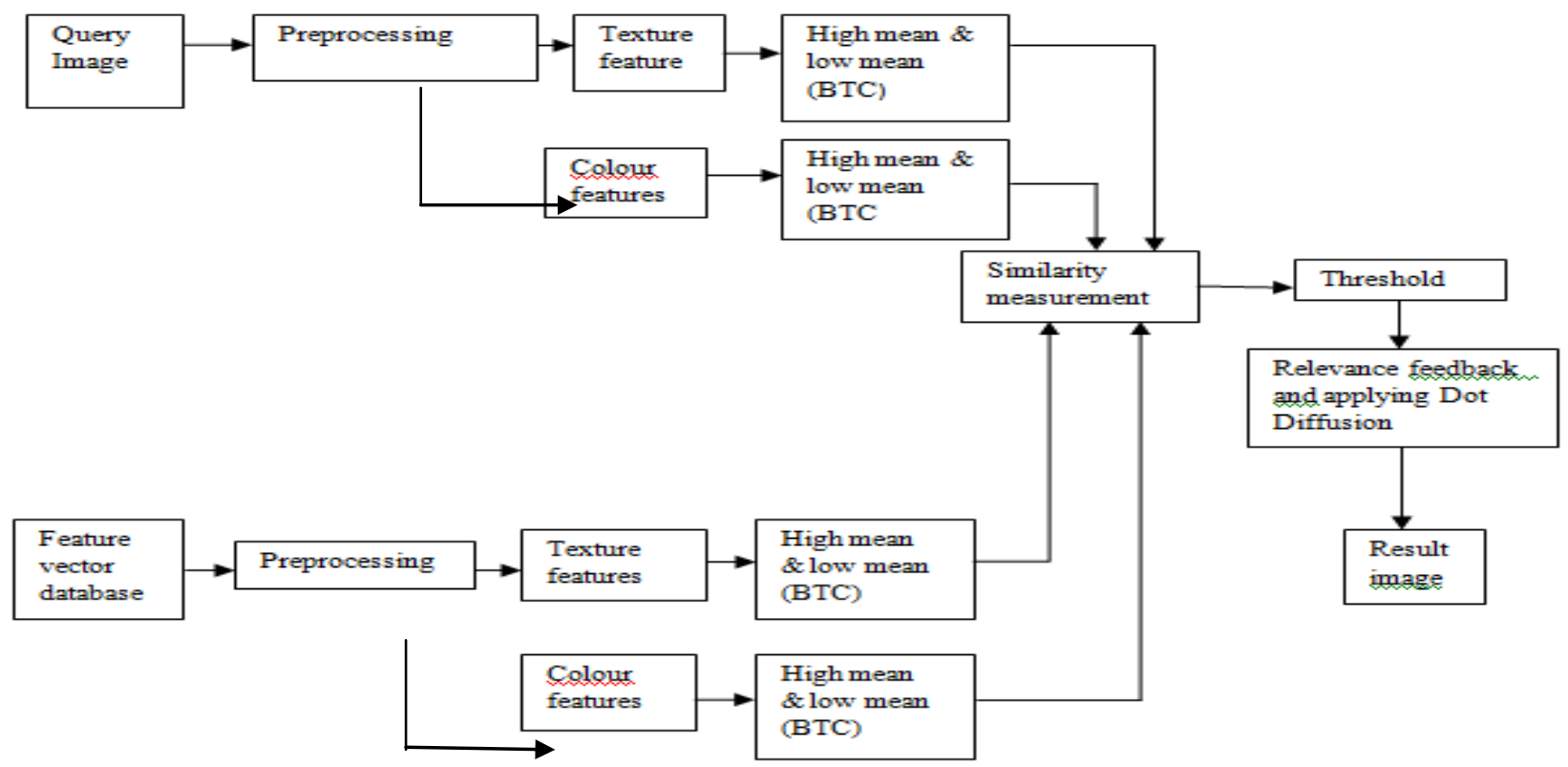

Fig 1: Proposed Architecture for satellite image retrieval using Dot-Diffusion Block Truncation Coding

\section{EXPERIMENTAL RESULTS}

The following are the performed results of Improved Block Truncation Coding Using Dot Diffusion for Satellite Image Retrieval. Image retrieval is carried out using the operating system associated with MATLAB (version 7.6) along with Hardware associated as follows: i) Processor: Intel core i3, ii) CPU Speed:3.20GHz, iii) OS: Windows 7 and iv) RAM: 4GB.

The outcome to the features like colour based retrieval and texture based retrieval using Improved Block Truncation Coding are shown below in Fig 2

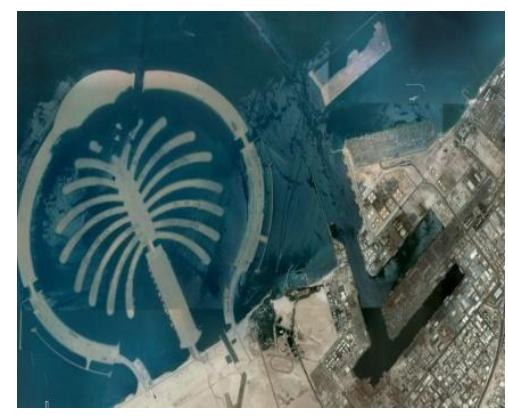

(i)
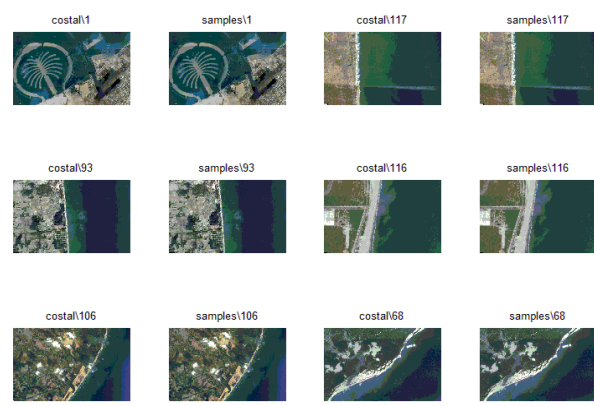

(ii)
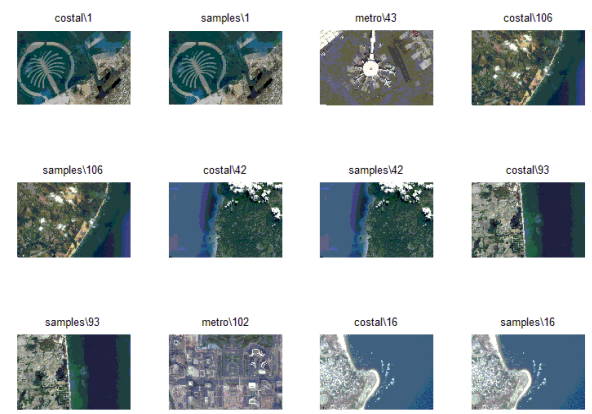

(iii)

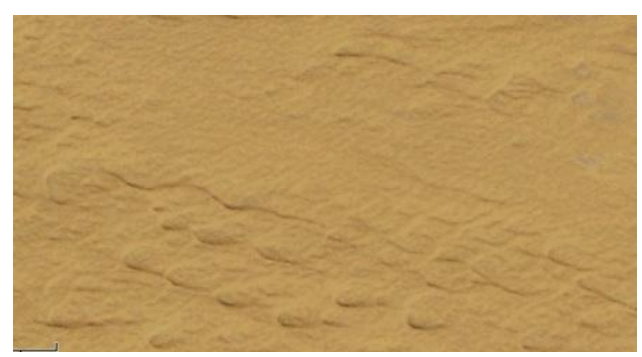

(i)
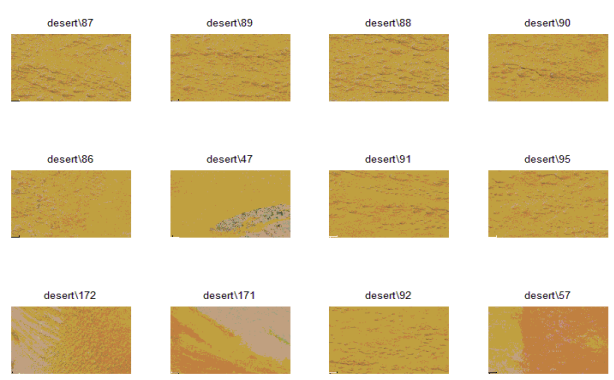

(ii) 


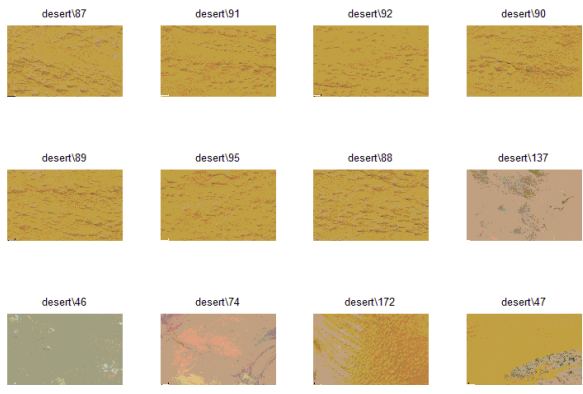

(iii)

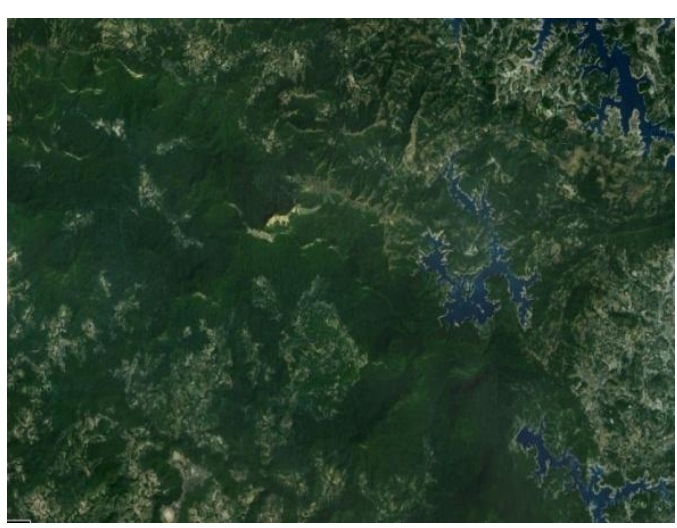

(i)
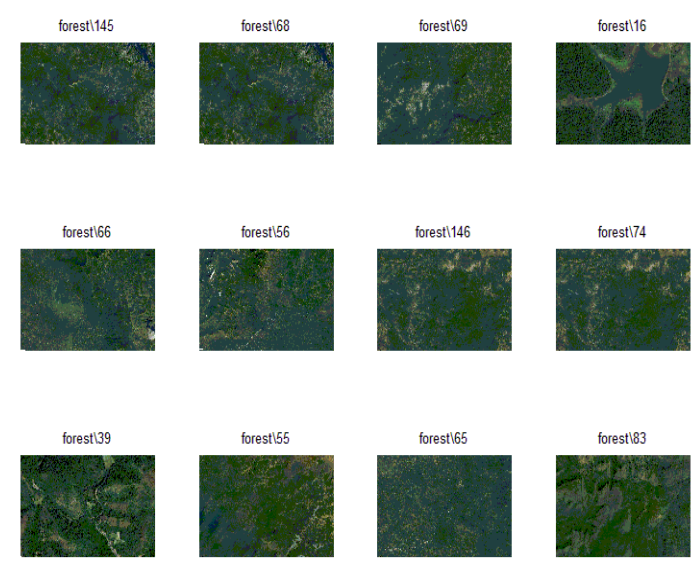

(ii)
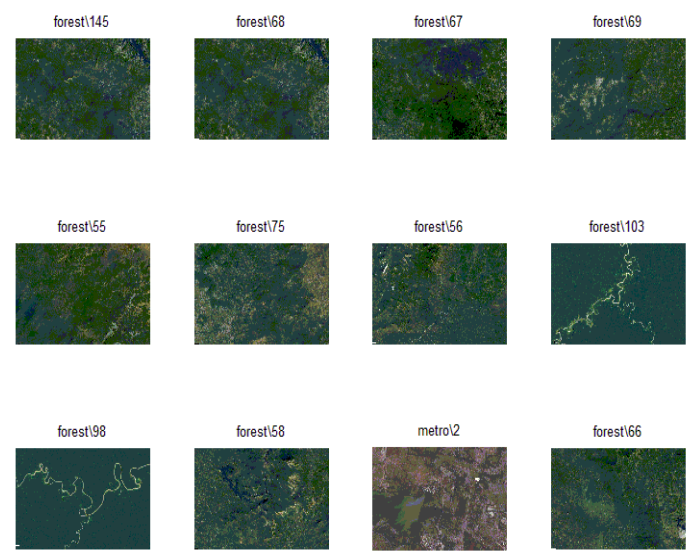

(iii)

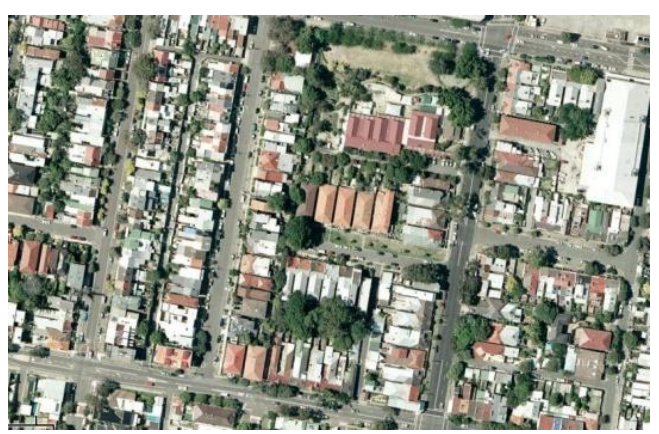

(i)
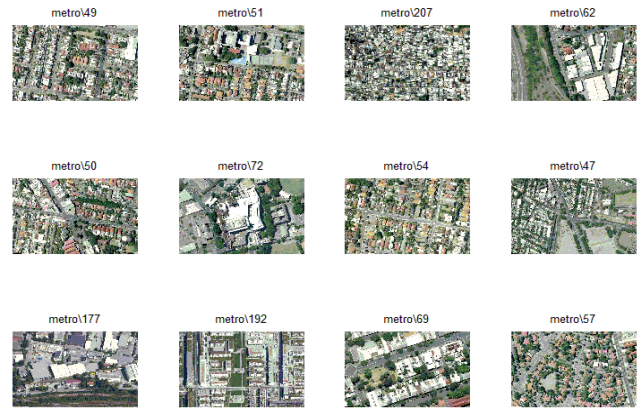

(ii)
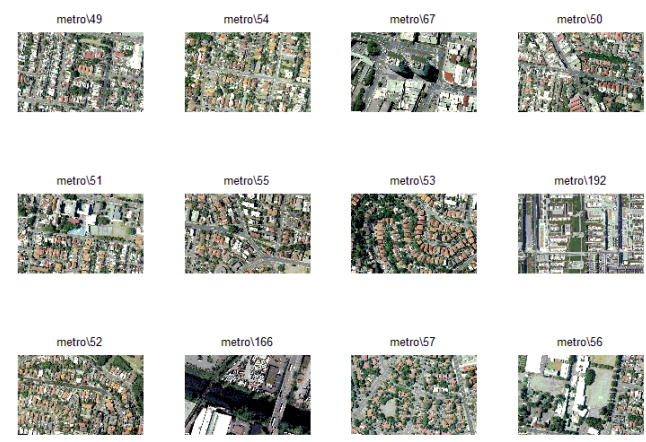

(iii)

Fig.2. Retrieved Results (i) Query Image (ii) Colour based extracted images (iii) Texture based extracted images

Performance Analysis

In this work, we have considered the actual databases of 152 satellite images. In this method 152 images are generally taken into consideration regarding instruction graphics and here 100 graphics are considered for screening images.

TABLE I

PERFORMANCE OF PROPOSED IMPROVED BLOCK TRUNCATION CODING USING DOT DIFFUSION AND THE EXISTING SYSTEM

SUCH AS BLOCK TRUNCATION CODING CLASSIFICATION RESULTS FOR COASTAL, METRO, FOREST, DESERT AREA IMAGES.

\begin{tabular}{|l|c|c|c|c|}
\hline \multirow{2}{*}{ Measures } & \multicolumn{2}{|c|}{ WATER BODY AREA } & \multicolumn{2}{c|}{ METRO AREA } \\
\cline { 2 - 5 } & $\begin{array}{c}\text { Proposed } \\
\text { DDBTC }\end{array}$ & BTC & $\begin{array}{c}\text { Proposed } \\
\text { DDBTC }\end{array}$ & BTC \\
\hline Reliability & 89 & 80.102 & 89.94 & 79.513 \\
\hline Sensitivity & 90 & 82.14 & 90.109 & 78.5 \\
\hline Specificity & 89.5 & 79.30 & 91.2 & 82.22 \\
\hline
\end{tabular}

(i) 


\begin{tabular}{|l|c|c|c|c|}
\hline \multirow{2}{*}{ Measures } & \multicolumn{2}{|c|}{ FOREST AREA } & \multicolumn{2}{c|}{ DESERT AREA } \\
\cline { 2 - 5 } & $\begin{array}{c}\text { Proposed } \\
\text { DDBTC }\end{array}$ & BTC & $\begin{array}{c}\text { Proposed } \\
\text { DDBTC }\end{array}$ & BTC \\
\hline Reliability & 87.2 & 86.33 & 85.1 & 79.521 \\
\hline Sensitivity & 90.14 & 85.11 & 89.14 & 85.80 \\
\hline Specificity & 88.9 & 83.1 & 87.56 & 89.15 \\
\hline
\end{tabular}

(ii)

Here, Table 1 explains the effectiveness of proposed Dot Diffusion Block Truncation Coding for satellite image retrieval .

\section{TABle II}

Proposed SYSTEM PERFORMANCE RETRIEVAL RESUlTS OF COSTAL AREA, DESERT AREA, FOREST AREA AND METRO IMAGES

\begin{tabular}{|c|c|c|c|c|c|c|}
\hline \multicolumn{7}{|c|}{ Proposed system } \\
\hline $\begin{array}{c}\text { Query } \\
\text { Images }\end{array}$ & \multicolumn{2}{|c|}{ BTC } & \multicolumn{2}{c|}{ COLOUR } & \multicolumn{2}{c|}{ TEXTURE } \\
\cline { 2 - 7 } $\begin{array}{c}\text { Precision } \\
\text { AREA }\end{array}$ & $63 \%$ & $62.58 \%$ & $50 \%$ & $50 \%$ & $50 \%$ & $50 \%$ \\
\hline $\begin{array}{c}\text { DESERT } \\
\text { AREA }\end{array}$ & $83.21 \%$ & $82.10 \%$ & $70 \%$ & $70 \%$ & $70 \%$ & $70 \%$ \\
\hline $\begin{array}{c}\text { FOREST } \\
\text { AREA }\end{array}$ & $82 \%$ & $82 \%$ & $82 \%$ & $82 \%$ & $70 \%$ & $70 \%$ \\
\hline $\begin{array}{c}\text { METRO } \\
\text { AREA }\end{array}$ & $98 \%$ & $98 \%$ & $96.41 \%$ & $95.1 . \%$ & $97 \%$ & $97 \%$ \\
\hline
\end{tabular}

Here, Table II displays precision and recall calculated for a dataset of 152 images.
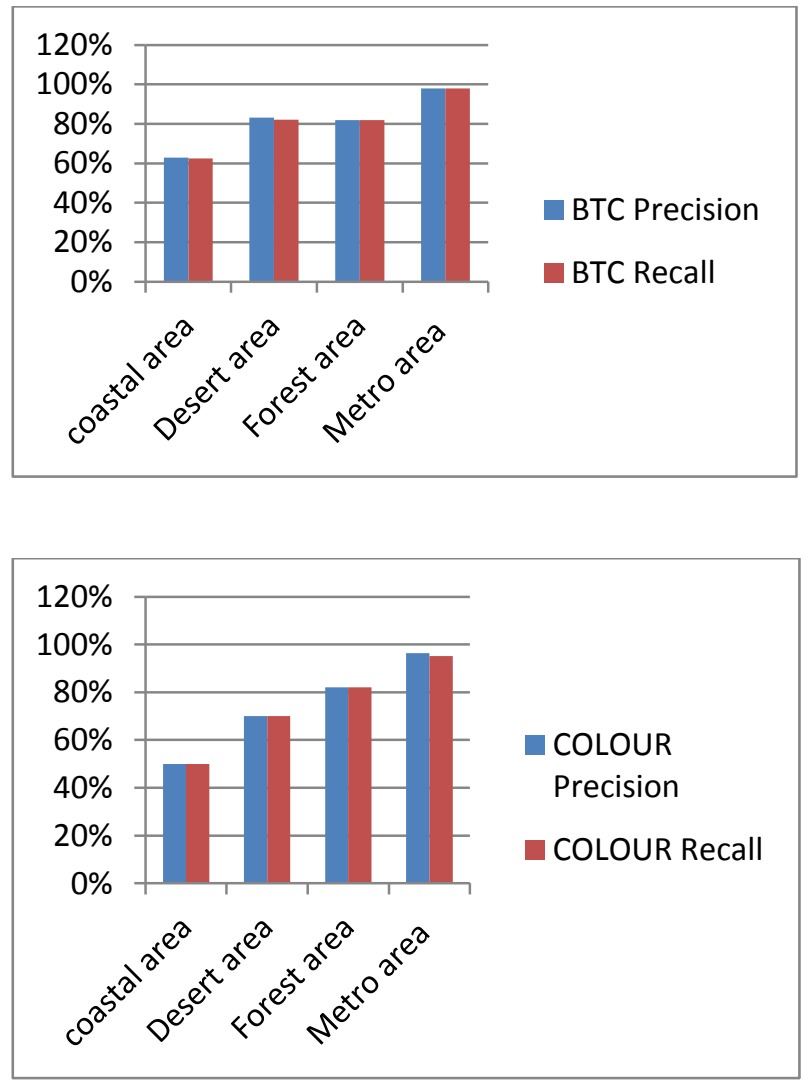

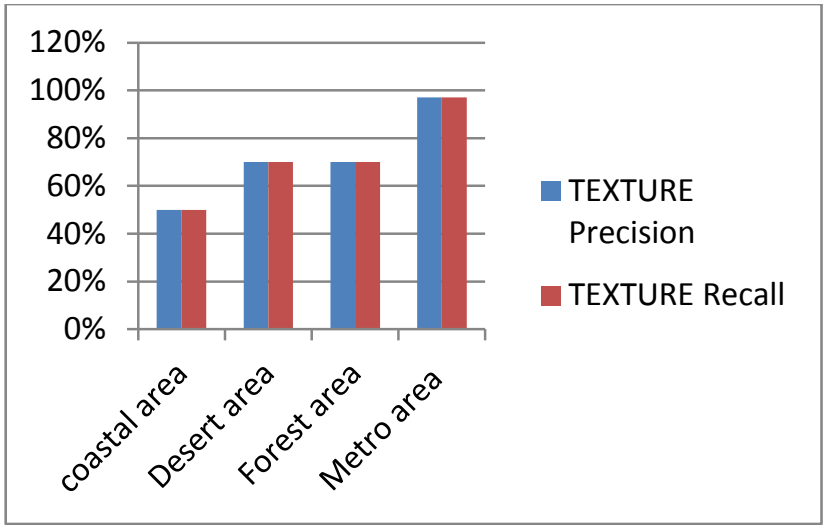

To calculate the precision and recall following formulas are used as mentioned in Table 3.

Precision $=($ images those are retrieved) $\cap$ (images those are relevant) / (images those are retrieved) .......................... (1.1)

Recall $=$ (images those are retrieved) $\cap$ (images those are relevant) / (images those are relevant)

In overall performance analysis metrics each of our proposed Dot Diffusion Block Truncation Coding for satellite image retrieval system offers high detail along with recall rate in comparison with the current BTC technique.

\section{ACKNOWLEDGMENTS}

Myself is deeply indebted to my Guide Kiran .A. Bhandari for his guidance and helpful suggestions during the research.

\section{CONCLUSION}

In this paper Dot Diffusion technique is suggested for the satellite images such as coastal, metro area, Forest and Desert which are widely-used for the performance evaluation. Proposed technique is found to attain $90 \%, 91 \%$, and $92 \%$ of reliability, sensitivity and specificity attributes respectively. Thus, in the performance evaluation it is proved that when compared with the existing systems; our proposed system retrieves the desired result images with accuracy .The future work can be by using other half toning methods like errordiffusion at relevance feedback.

\section{REFERENCES}

[1] M. Mese and P. P. Vaidyanathan, "Optimized half toning using dot diffusion and methods for inverse half toning," IEEE Trans. Image Processing, vol. 9, no. 4, pp. 691709, Apr. 2000

[2] G. Rafiee, S.S. Dlay, and W.L. Woo," A Review of Content-Based Image Retrieval", IEEE, 2010. Tavel, P. 2007 Modeling and Simulation Design. AK Peters Ltd Tavel, P. 2007 Modeling and Simulation Design. AK Peters Ltd.

[3] Kekre H.B., Bharadi, V.A., Thepade S.D. , Mishra B.K., Ghosalkar, S.E., Sawant S.M. , "Content Based Image Retrieval Using Fusion of Gabor Magnitude and Modified Block Truncation Coding", IEEE computer society, 2010 IEEE.

[4] Dr.H.B.Kekre, S.D. Thepade et al.,"Image Retrieval with Shape Features Extracted using Gradient Operators and Slope Magnitude Technique with BTC",International Journal of Computer Applications (0975 - 8887) Volume 6- No.8, September 2010 Brown, L. D., Hua, H., and 
Gao, C. 2003. A widget framework for augmented interaction in SCAPE.

[5] Y N Mamatha and A.G Ananths and S 0 Neil, "Content Based Image Retrieval of Satellite Imageries Using Soft Query Based Color Composite Techniques",IEEE Trans on Acoustic speech signal processing, Vol I, No.3, pp. 1278- 1288,1986

[6] Yu-f-x, Luo, H, Lu, Z-m"Colour image retrieval using pattern co-occurrence matrices based on BTC and VQ"IET Electronic Letters (Volume:47, Spector, A. Z. 1989. Achieving application requirements. In Distributed Systems, S. Mullender

[7] Purohit Shrinivasacharya, Dr. M. V Sudhamani"Content Based Image Retrieval System using Texture and Modified Block Truncation Coding"2013 International Conference on Advanced Computing and Communication Systems (ICACCS -2013), Dec. 1921,2003
[8] Delp, E. J., Saenz, M., and Salama, P., Block Truncation Coding(BTC),"Handbook of Image Bovik A.C ,Academic Press , pp. 176-181, 2000

[9] Dr. Fuhui Long, Dr. Hongjiang Zhang and Prof. David Dagan Feng, "Fundamentals of Content-Based Image Retrieval

[10] H B Kekre and V A Bharadi, "Modified BTC \& Walsh coefficients Based Features for Content Based Image Retrieval" NCICT, India

[11] H.B.Kekre, S.D.Thepade "Image Retrieval using Augmented Block Truncation Coding Techniques" International Conference on Advances in Computing, Communication and Control (ICAC3'09)

[12] E. J. Delp and O. R. Mitchell, "Image compression using block truncation coding," IEEE Trans. Communication., vol. 27, no. 9,pp. 1335-1342, Sep. 1979 